International Journal of Foundations of Computer Science

Vol. 15 No. 4 (2004) 685

(C) World Scientific Publishing Company

\title{
ERRATUM
}

\section{ON THE MINIMAL POLYNOMIAL OF A MATRIX}

\author{
[IJFCS, Vol. 15, No. 1 (2004) 89-105]
}

Thanh Minh Hoang and Thomas Thierauf

The page below was inadvertently omitted from the published paper, and should be inserted between page 96 and page 97 .

Let $a_{1}=\left(A_{1}^{m}\right)_{1, n_{1}}$ and $a_{2}=\left(A_{2}^{l}\right)_{1, n_{2}}$. As explained in the first part of the proof, (in logspace) we can compute matrices $B_{1}$ and $B_{2}$ such that

$$
\begin{aligned}
& \mu_{B_{1}}(x)=x^{2 m+2}-a_{1} x^{m+1}, \\
& \mu_{B_{2}}(x)=x^{2 l+2}-a_{2} x^{l+1} .
\end{aligned}
$$

By $C$ we denote again the companion matrix of $x^{2 m+2}$. Define the matrix

$$
D=\left[\begin{array}{ccc}
B_{1} & \mathbf{0} & \mathbf{0} \\
\mathbf{0} & B_{2} & \mathbf{0} \\
\mathbf{0} & \mathbf{0} & C
\end{array}\right]
$$

Then we get

$$
\begin{aligned}
\mu_{D}(x) & =\operatorname{lcm}\left\{\mu_{B_{1}}(x), \mu_{B_{2}}(x), \mu_{C}(x)\right\} \\
& =\operatorname{lcm}\left\{x^{m+1}\left(x^{m+1}-a_{1}\right), x^{l+1}\left(x^{l+1}-a_{2}\right), x^{2 m+2}\right\} \\
& =x^{2 m+2} \operatorname{lcm}\left\{x^{m+1}-a_{1}, x^{l+1}-a_{2}\right\} .
\end{aligned}
$$

Since $m>l$, we have

$$
\operatorname{deg}\left(\mu_{D}(x)\right)= \begin{cases}2 m+l+3, & \text { for } a_{1}=0, a_{2} \neq 0, \\ 3 m+3, & \text { for } a_{1} \neq 0, a_{2}=0, \\ 2 m+2, & \text { for } a_{1}=0, a_{2}=0, \\ 3 m+3+r, & \text { for } a_{1} \neq 0, a_{2} \neq 0, \text { where } r>0 .\end{cases}
$$

We concluded that for every $w$

$$
\begin{aligned}
w \in L & \Longleftrightarrow a_{1}=0 \text { and } a_{2} \neq 0 \\
& \Longleftrightarrow \operatorname{deg}\left(\mu_{D}(x)\right)=2 m+l+3 .
\end{aligned}
$$

This completes the proof of the theorem.

By Proposition 1 and Theorem 1 we obtain the following corollary.

Corollary 1 1) DegMinPol $\leq$ is complete for $\mathbf{C}_{=} \mathbf{L}$.

2) $\mathrm{DegMinPoL}_{=}$is complete for $\mathbf{C}_{=} \mathbf{L} \wedge \mathbf{c o C}_{=} \mathbf{L}$.

The following lemma completes the proof of Theorem 1 .

Lemma 1 Let $A$ be an $n \times n$ matrix and let $m \geq 1$. For any $k \geq 1$ there is a matrix $\widetilde{A}$ of order $p=n(m k+1)$ such that $\left(A^{m}\right)_{1, n}=\left(\widetilde{A}^{k m}\right)_{1, p}$. 